Седова Ольга Петровна, доцент кафедры «Перерабатывающие технологии и продовольственная безопасность» ФГБОУ ВО «Волгоградский государственный аграрный университет» (400002, Волгоград, пр-т Университетский, 26), кандидат сельскохозяйственных наук, ORCID: 0000-0001-99368558. E-mail: o.p.sedova@yandex.ru.

Гурба Алексей Валерьевич, преподаватель-исследователь ФГБОУ ВО «Волгоградский государственный аграрный университет» (400002, Волгоград, пр-т Университетский, 26), ORCID: 00000002-0557-9950. E-mail: algurru@mail.ru.

DOI: $10.32786 / 2071-9485-2021-02-45$

\title{
RESEARCH COMPLEX FOR STUDYING THE INFLUENCE OF HIGH-VOLTAGE OF ELECTRIC IMPULSE EFFECTS ON LARVAE OF LOCUST INSECTS
}

\author{
I.V. Yudaev', V.A. Eviev' ${ }^{2}$, B.I. Belyaeva ${ }^{2}$, R.G. Kokurin ${ }^{3}$ \\ ${ }^{1}$ Federal State Budget Educational Institution of Higher Education \\ "St. Petersburg State Agrarian University» \\ ${ }^{2}$ Federal State Budget Educational Institution of Higher Education \\ "Kalmyk State University named after B. Gorodovikov» \\ ${ }^{3}$ Azov-Black Sea Engineering Institute - the branch of Federal State Budget Educational Institution \\ of Higher Education «Don State Agrarian University» in Zernograd
}

Received 05.03.2021

Submitted 11.05.2021

Introduction. For centuries, locust insects have been causing harm to the crop sector of agriculture, which is estimated as significant in more than 60 countries of the world. Today, when the problem of providing food to the world's population is more acute than ever, it is necessary not only to improve the culture of agriculture, to develop environmentally friendly technologies for the production of high-quality products, but it is also necessary to look for new approaches in the fight against harmful insects and diseases that would be environmentally safe and did not contain harmful components in the products. Therefore, in our opinion, the most interesting from the point of view of high technological efficiency, environmental and food safety is the option of locust destruction using high voltage of electrical impulses. The use of electric impulse technologies can be implemented as an effective agricultural technique for combating larvae (egg capsules) of locust insects. Object. The object of the research is designed impulse voltage generator, which is the main element of experimental research complex for studying the effect of high-voltage of electrical impulses on locust larva located in the soil layer. Materials and methods. To study the effect of high voltage electrical impulses on the egg-pods, it is necessary to conduct series of experimental studies in laboratory to determine the parameters of such an effect and identify processing modes. The main unit of the research facility is impulse voltage generator. Its work determines the effectiveness of the impact, the possibility of stable work, the formation of safety requirements and the features of its operation. Results and conclusion. The designed and manufactured impulse voltage generator has been tested and verified in operation, showing high stability under laboratory conditions as part of experimental research complex. The research setup allows, depending on the value of the processing voltage and the capacitance of the output stage capacitor: 1) to set the impulse repetition rate, the upper limit of the values of which depends on the previously entered data, and the lower one is $1 \mathrm{~Hz} ; 2$ ) to set counter for the number of impulses, which executing preset algorithm controls their generation; 3 ) to generate high-voltage impulses with amplitude of 16 $\mathrm{kV}$ with exponential cut and of various duration. The research complex, assembled on the basis of impulse voltage generator, allows not only the processing of soil samples under laboratory conditions, but also makes it possible to process the experimental results in the function of continuous scanning using computer, making adjustments to the processing and evaluating the obtained results.

Key words: larvae (egg capsules) of locust insects; high voltage of electrical impulses; pulse voltage generator; design and testing; research experimental set-up.

Citation. Yudaev I.V., Eviev V.A., Belyaeva B.I., Kokurin R.G. Research complex for studying the influence of high-voltage of electric impulse effects on larvae of locust insects. Proc. of the Lower Volga AgroUniversity Comp. 2021. 2(62). 454-467 (in Russian). DOI: 10.32786/2071-9485-2021-02-45. 


\title{
***** H3BECTHЯ ***** \\ НИЖНЕВОАЖСКОГО АГРОУНИВЕРСИТЕТСКОГО КОМПАЕКСА: \\ НАУКА И ВЫСШЕЕ ПРОФЕССИОНААЬНОЕ ОБРАЗОВАНИЕ
}

Author's contribution. All authors of this research paper have directly participated in the planning, execution, or analysis of this study. All authors of this paper have read and approved the final version submitted.

Conflict of interest. The authors declare no conflict of interest.

УДК 362.727

\section{ИССЛЕДОВАТЕЛЬСКИЙ КОМПЛЕКС ДЛЯ ИЗУЧЕНИЯ ВЛИЯНИЯ ВЫСОКОВОЛЬТНЫХ ЭЛЕКТРОИМПУЛЬСНЫХ ВОЗДЕЙСТВИЙ НА ЛИЧИНКИ САРАНЧОВЫХ НАСЕКОМЫХ}

\author{
И. В. Юдаев ${ }^{1}$, доктор технических наук, профессор \\ В. А. Эвиев ${ }^{2}$, доктор технических наук, профессор \\ Б. И. Беляева ${ }^{2}$, кандидат педагогических наук, доиент \\ Р. Г. Кокурин ${ }^{3}$, аспирант \\ ${ }^{I}$ ФГБОУ ВО «Санкт-Петербургский государственный аграрный университет» \\ ${ }^{2}$ ФГБОУ ВО «Калмыцкий государственный университет им. Б. Городовикова» \\ ${ }^{3}$ Азово-Черноморский инженерный институт - \\ филиал ФГБОУ ВО «Донской государственный аграрный университет», г. Зерноград
}

Дата поступления в редакцию 05.03.2021

Дата принятия к печати 11.05.2021

Актуальность. Саранчовые насекомые уже не одно столетие наносят вред растениеводческой отрасли сельского хозяйства, который оценивается как существенный на территории более чем 60 стран мира. Сегодня, когда проблема с обеспечением продуктами питания населения Земли стоит как никогда остро, необходимо не только повышать культуру земледелия, развивать экологически безопасные технологии производства качественной продукции, но и искать новые подходы в борьбе с вредными насекомыми и болезнями, которые были бы экологически безопасными и не содержали вредных компонентов в продуктах. Поэтому, на наш взгляд, наиболее интересным с точки зрения высокой технологической эффективности, экологической и пищевой безопасности является вариант уничтожения саранчи при помощи электрических импульсов высокого напряжения. Применение электроимпульсных технологий может быть реализовано как эффективный агроприем для борьбы с личинками (кубышками) саранчи. Объект. Объектом исследования является спроектированный генератор импульсного напряжения, который является основным элементом экспериментального исследовательского комплекса для изучения влияния электроимпульсных высоковольтных воздействий на личинки саранчи, находящиеся в почвенном слое. Материалы и методы. Для изучения влияния воздействия на кубышки электрических импульсов высокого напряжения необходимо провести серию экспериментальных исследований в условиях лаборатории для выяснения параметров такого воздействия и выявления режимов обработки. Основным блоком установки для проведения исследований является генератор импульсного напряжения. Его работа определяет эффективность воздействия, возможность устойчивой работы, формирование требований безопасности и особенностей его эксплуатации. Результаты и выводы. Спроектированный и изготовленный генератор импульсного напряжения испытан и проверен в работе, показал в лабораторных условиях высокую стабильность в составе экспериментального исследовательского комплекса. Исследовательская установка позволяет в зависимости от значения напряжения обработки и емкости конденсатора выходного каскада: 1) задавать частоту следования импульсов, верхняя граница значений которых зависит от предварительно введённых данных, а нижняя - 1 Гц; 2) устанавливать счётчик количества импульсов, который при отработке предварительно заданного алгоритма управляет их генерированием; 3) генерировать высоковольтные импульсы с амплитудой 16 кВ с экспоненциальным срезом и различной длительности. Исследовательский комплекс, собранный на основе генератора импульсного напряжения, позволяет в лабораторных условиях не только осуществлять обработку почвенных образцов, но и дает возможность в функции непрерывного сканирования с помощью компьютера обрабатывать результаты экспериментов, внося коррективы в обработку и оценивая получаемые результаты. 
Ключевые слова: личинки саранчовых насекомых, электрические импульсы высокого напряжения, генераторы импульсного напряжения, электроимпульсные технологии.

Цитирование. Юдаев И. В., Эвиев В. А., Беляева Б. И., Кокурин Р. Г. Исследовательский комплекс для изучения влияния высоковольтных электроимпульсных воздействий на личинки саранчовых насекомых. Известия НВ АУК. 2021. 2(62) 454-467. DOI: 10.32786/2071-9485-2021$02-45$.

Авторский вклад. Все авторы настоящего исследования принимали непосредственное участие в планировании, выполнении или анализе данного исследования. Все авторы настоящей статьи ознакомились и одобрили представленный окончательный вариант.

Конфликт интересов. Авторы заявляют об отсутствии конфликта интересов.

Введение. При выращивании продукции сельского хозяйства и ее последующей переработке на предприятиях и комплексах интенсивно и с высокими показателями эффективности применяют разнообразные электротехнологические операции, реализуемые при помощи специально изготовленных установок. Из большого разнообразия реализации электрофизических воздействий на объекты растительного происхождения, электроимпульсное подведение электрической энергии к последним считается не только безопасным с точки зрения пищевых и экологических аспектов, но и осуществляемым с высокими показателями технологической эффективности и минимальными затратами энергии на операцию или процесс $[2,6,10,11,13]$.

Отдельные варианты электроимпульсных технологий при обработке направлены на достижение летальных исходов, как в случае уничтожения летающих насекомых, или организации необратимого повреждения внутриклеточных структур растительных объектов, как в случае борьбы с сорняками, прореживания посевов и посадок, электроплазмолиза растительного сырья, предуборочной подработки возделываемых растений и т.п. $[5,10,12,14]$.

На возможность использования электрических воздействий летальных доз для борьбы с вредителями, вредными насекомыми и болезнями, распространяемыми в почве под посевами и посадками сельскохозяйственных культур, обратили внимание еще в XVIII веке. Сегодня эти исследования как никогда актуальны, и они продолжаются в лабораториях и научных центрах, позволяя получать новые сведения о механизмах необратимого повреждения биологических тканей насекомых, разрабатывать новые технические средства, совершенствовать применяемые электротехнологии, снижая затраты совокупной энергии и не нанося урона окружающей среде.

Одной из первых конструкций для реализации варианта уничтожения при помощи электричества насекомых и их личинок, обитающих в поверхностном слое почвы, можно считать описанный в 1903 году «электрический снаряд для массового истребления вредных для сельского хозяйства насекомых» системы русского инженераэлектротехника 3. Локуцеевского.

Более подробно решением этой проблемы с обоснованием и экспериментальной проверкой как в лабораторных, так и полевых условиях занимались с 1936 по 1939 годы ученые Всесоюзного НИИ лесного хозяйства (А. В. Луговой, С. Я. Турлыгин, Д. С. Беклемышев). Эта группа исследователей установила, что для борьбы с такими насекомыми, как личинки майского жука (хруща), наиболее эффективно использовать «импульсную форму приложения энергии» характеризующуюся следующими параметрами: амплитуда импульса напряжения - 4,0-9,0 кВ; градиент электрического поля - 0,32,5 кВ/см; количество воздействующих импульсов - 1-10 шт.; постоянная времени разряда $-1,0 \cdot 10^{-2}$ сек; емкость разрядного контура - до $12,0 \cdot 10^{-6} \Phi$. 
В 1954-1956 годах сотрудники Института биологической физики АН СССР (А. А. Передельский, Л. С. Осипова, В. Н. Ефимов) изучали применение импульсов высокого напряжения для борьбы со свекловичным долгоносиком и экспериментально выявили следующие эффективные режимы его необратимого травмирования, приводящего к летальному исходу: градиент электрического поля - 1,0-3,5 кВ/см; количество воздействующих импульсов - 1-12 шт.; постоянная времени разряда $-(0,2-1,0) \cdot 10^{-3}$ сек; емкость разрядного контура - $(0,13-1,39) \cdot 10^{-6} \Phi$; плотность повреждающего тока - 0,3-0,68 A/cm².

В 1956-1958 годах сотрудниками Всесоюзного НИИ электрификации сельского хозяйства (М. Г. Евреинов, И. С. Смирнова, Л. С. Лурье, А. А. Тильвикас) был сконструирован и изготовлен пилотный образец мобильного генератора, который позволял осуществлять обработку почвы при борьбе с вредителями и обитающими в ней насекомыми и работал на следующих режимах: градиент электрического поля - 1,4-2,15 кВ/см; частота следования импульсов - 7-30 импульсов в секунду; постоянная времени разряда - $(0,01-0,14) \cdot 10^{-3}$ сек; емкость разрядного контура - $(0,32-1,54) \cdot 10^{-6} \Phi$.

В 70-х годах XX века в Тимирязевской сельскохозяйственной академии исследователем В. Т. Скляром изучалось влияние высоковольтных импульсных воздействий на хлебных жужелиц, клопов-черепашек и слизней сетчатых голых, результатом которого стали выявленные режимы их электрического необратимого повреждения. Так, энергоемкость летального импульса на один исследуемый объект составила: для хлебных жужелиц - 1,4 Дж; для слизней - 1,2 Дж; для клопов-черепашек - 1,1 Дж. Для уничтожения галловой нематоды и гриба-возбудителя капустной килы удельный расход электрической энергии соответственно составил 24,7 и 27,8 кВт·ч/ ${ }^{3}$.

Отдельный вопрос, лежащий на поверхности, касается влияния электромагнитных воздействий на микроорганизмы [15], в том числе влияния воздействующих высоковольтных импульсов на почвенную биоту [9]. Проведенные полевые исследования [4] показали, что конкретные значения параметров импульсных воздействий и видовой состав почвенных микроорганизмов, на которые оказываются воздействия, приводят как к угнетению жизнедеятельности последних, так и к их стимуляции. Экспериментально было доказано, что характер и степень электрообработки находятся в прямой зависимости от количества влаги в почвы. Низкая влажность почвы (10-12 \%) способствует реализации эффекта стимуляции почвенной микрофлоры с четко проявляющимся эффектом последействия, при высоком насыщении почвы влагой (18 \%) наблюдается растянутый по времени эффект угнетения. Воздействие электрических импульсов на почвенный объем позволяет увеличить, за счет эффекта последействия, подвижные соединения таких элементов, как калий и фосфор, на 1-2 мг/кг почвы, прибавив тем самым урожай культур, например, зерновых на 0,1-0,12 ц/га. Такая обработка приводит и к увеличению содержания гумуса в почве - 7-9 \%, при его первоначальном содержании 2,65 \%. Стимуляция жизнедеятельности почвенной микрофлоры наблюдалась на самых их неприхотливых представителях - олигонитрофильных бактериях, деятельность которых увеличивала усвояемые формы азота в почве. При этом обработку «не следует вести на «жестких» режимах, характеризующихся большой плотностью энергии» [4].

В настоящее время возникла острая необходимость поиска экологически чистых и малоэнергозатратных способов борьбы с наиболее вредоносным насекомым - саранчой, что продиктовано отсутствием действенных мероприятий и наличием у существующих способов негативного воздействия, прежде всего, из-за ядохимикатов на окружающую среду и биоту почвы.

Самыми вредоносными видами саранчи являются перелётные: мароккская и азиатская саранча, итальянский прус и сибирская кобылка. Специалисты продовольственной и сельскохозяйственной организации ООН (ФАО) подсчитали, что «одна стая саранчи, насчитывающая до 80 млн особей, в день съедает столько же, сколько могут 
съесть 35 тыс. человек. Эти стаи могут перемещаться со скоростью 15-20 км/ч до 20 ч, достигая размера облака - 10 км в ширину и до 200 км в длину». Экономическим порогом вредоносности по количеству личинок на квадратный метр считается для: итальянского пруса и мароккской саранчи - 2-5; перелетной азиатской саранчи - 1-2; по не стадным саранчовым (кобылкам) - 10-15, в то время как одна женская особь за свою жизнь откладывает в среднем до 1,5 тысяч личинок.

В качестве достаточно новых и пока еще редко используемых способов борьбы рассматриваются различные физические воздействия, летальные для насекомых - электрические, магнитные и электромагнитные поля; КВЧ и СВЧ-энергия; концентрированное излучение и т.п. Перспективным способом уничтожения саранчовых насекомых, на наш взгляд, следует считать применение высоковольтных импульсных воздействий летальной дозы, которое будет эффективным как при необратимом повреждении яйцекладки саранчи в почве, так и при уничтожении взрослых особей $[1,3]$.

Реализовать на практике процесс электроимпульсного воздействия на личинки, расположенные в почве, и на взрослых насекомых, перемещающихся по поверхности почвы, не представляется возможным без специально спроектированной электротехнологической установки, с помощью которой можно формировать и подводить к объектам обработки импульсы высокого напряжения требуемой энергии, амплитуды, длительности, необходимой формы и частоты их следования $[7,8,16]$.

Целью представленного в статье научно-практического исследования является обоснование особенностей построения структурной и принципиальной схемы генератора импульсного напряжения, являющегося основным элементом экспериментального исследовательского комплекса для изучения влияния электроимпульсных высоковольтных воздействий на личинки саранчи, находящиеся в почвенном слое.

Материалы и методы. Для выявления технологически эффективных параметров и режимов электроимпульсной обработки личинок саранчи или так называемых кубышек, откладываемых в почве на небольшой глубине взрослыми самками, а также для изучения сущности механизма электроимпульсного необратимого повреждения этих кубышек необходимо провести лабораторные исследования и полевые опыты. Реализовать такие всесторонние исследования невозможно без использования специальных электротехнологических установок, основным структурным компонентом которых является блок формирования импульсов высокого напряжения, или генератор высоковольтных импульсов. Этот блок должен осуществлять настройку и регулировку параметров импульсов, формируемых на его выходе, с необходимой точностью; обеспечивать высокую надежность в работе; характеризоваться небольшой массой и габаритами; быть простым в сборке, наладке и эксплуатации; обладать высокой степенью электробезопасности при функционировании. Обратив внимание на все перечисленные выше требования, решили спроектировать и изготовить генератор импульсного напряжения с емкостным накопителем энергии в выходном (разрядном) каскаде, который позволял бы получать высоковольтные импульсы с крутым фронтом, экспоненциальным срезом и регулируемой амплитудой импульса.

Результаты и обсуждение. Разрабатываемый генератор является основным компонентом лабораторного исследовательского комплекса для изучения влияния электрических импульсов высокого напряжения на разнообразные объекты, в том числе на объекты растительного происхождения, почву, личинки вредных насекомых и т.п. Генератор импульсного напряжения состоит из основных структурных элементов: блока управления, блока коммутации, а также высоковольтного блока. В блоке управления смонтирован регулирующий элемент настройки - задатчик поджигающих импульсов, 
или задатчик частоты следования воздействующих импульсов, который необходим для управления импульсным тиратроном, который смонтирован в блоке коммутатора. Рассмотрим принцип работы перечисленных блоков и их элементную базу (рисунок 1).
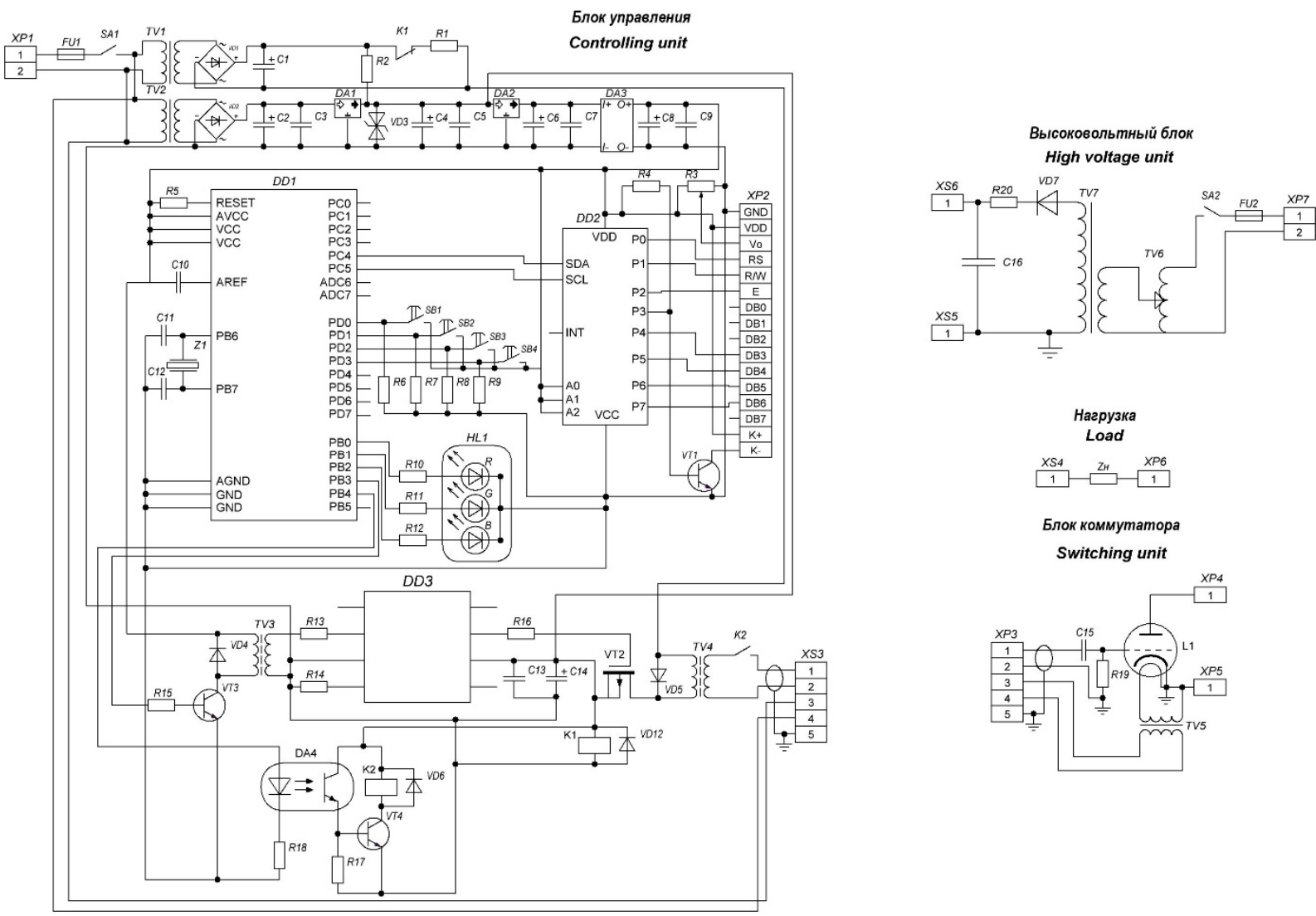

Рисунок 1 - Принципиальная электрическая схема генератора импульсного напряжения: C1 - 220 мкФ; С2,C4,C6,C8 - 1000 мкФ; С3,C5,C7,C9 - 330 нФ; С10 - 0.1 мкФ; С11,C12 - 15 пФ; C13 - 10 нФ; C14 - 10 мкФ; C15 -2200 пФ; R1,R3,R17 - 10 кОм; R2 - 15 Oм; R4 - 4.7 кОм;

R5,R13,R14,R15 - 1 кОм; R6,R7,R8,R9 - 100 кОм; R10,R11,R12,R18 - 2.7 кОм; R16 - 10 Oм;

R19 - 5 кОм; VD1,VD2 - BD610; VD3 - 1.5KE15A; VD4,VD5,VD6 - HER308; DA1 L7812ABV; DA2 - L7805ABV; DA3 - TMV 0505D; DA4 - EL817; DD1 - ATmega328; DD2 PCF8574T; DD3 - IR4428; VT1,VT3 - BD139; VT2 - 2SJ585; TV1 - 220/140; TV2 - 220/12; TV3 - 1:1;TV4 - МИТ-4B; TV5 - TH60-220-50; HL1 - BL-L515RGBW-CC; L1 - ТГИ2-400/16;

Figure 1 - Schematic diagram of impulse voltage generator: $\mathrm{C} 1-220 \mu \mathrm{F}$; $\mathrm{C} 2, \mathrm{C} 4, \mathrm{C} 6, \mathrm{C} 8-1000 \mu \mathrm{F}$; $\mathrm{C} 3, \mathrm{C} 5, \mathrm{C} 7, \mathrm{C} 9-330 \mathrm{nF} ; \mathrm{C} 10-0.1 \mu \mathrm{F} ; \mathrm{C} 11, \mathrm{C} 12-15 \mathrm{pF} ; \mathrm{C} 13-10 \mathrm{nF} ; \mathrm{C} 14-10 \mu \mathrm{F} ; \mathrm{C} 15-2200 \mathrm{pF}$; R1,R3,R17 - $10 \mathrm{k} \Omega ; \mathrm{R} 2-15 \Omega ; \mathrm{R} 4-4.7 \mathrm{k} \Omega$; R5,R13,R14,R15 - $1 \mathrm{k} \Omega ; \mathrm{R} 6, \mathrm{R} 7, \mathrm{R} 8, \mathrm{R} 9$ - $100 \mathrm{k} \Omega$; R10,R11,R12,R18 - $2.7 \mathrm{k} \Omega$; R16 - $10 \Omega$; R19 - 5 k $\Omega$; VD1,VD2 - BD610; VD3 - 1.5KE15A; VD4,VD5,VD6 - HER308; DA1 - L7812ABV; DA2 - L7805ABV; DA3 - TMV 0505D; DA4 EL817; DD1 - ATmega328; DD2 - PCF8574T; DD3 - IR4428; VT1,VT3 - BD139; VT2 - 2SJ585;

TV1 - 220/140; TV2 - 220/12; TV3 - 1:1;TV4 - MUT-4B; TV5 - TH60-220-50; HL1 - BLL515RGBW-CС; L1 - ТГИ2-400/16;

I. Блок управления. Для электрического питания этого блока сетевое переменное напряжение 220 В подводится к выводам первичных обмоток понижающих трансформаторов TV1 (220/140) и TV2 (220/12), в цепи которых размещен защитный предохранитель FU1. Со вторичных обмоток этих же трансформаторов напряжение снимается уже с уровнем в 140 и 12 В и подается на выпрямительные диодные мосты VD1 и VD2, укомплекто- 
ванные сглаживающими конденсаторами C1-C3. Резистор R1 обеспечивает разряд конденсатора C1, в том случае если блок управления генератора импульсного напряжения отключен от сети. При подключении блока управления к сети на обмотку реле К1 подается напряжение 12 В, и его контакт, размыкаясь, отключает резистор R1, прекращая тем самым замыкать конденсатор $\mathrm{C} 1$. Большая часть схемы запитывается стабилизированным напряжением 12 B, для чего используется линейный стабилизатор DA1 (L7812ABV). Гальваническая развязка организуется микросхемой DA3 (TMV0505D), которая в свою очередь запитывается стабилизированным напряжением 5 В от микросхемы DA2 (L7805ABV). Микроконтроллер DD1 (ATmega 328), размещенный в гальванически развязанной части схемы, отвечает за генерирование высоковольтных импульсов и контролирует состояние лампы L1 (импульсный тиратрон ТГИ2-400/16). Параметры эффективной электроимпульсной обработки задаются с использованием кнопочных элементов SB1-SB4.

Применение трехцветного светоизлучающего диода HL1 (BL-L515RGBW-CC) позволяет визуально контролировать реальное текущее состояние генератора импульсного напряжения. Синий цвет светодиода HL1 характеризует степень нагрева катода, который готов к работе по истечении 15 минут, в соответствии с паспортными данными, и именно эта продолжительность по времени определяет невозможность сразу же после подключения генератора к сети начать генерацию импульсов. Зеленый цвет светодиода HL1 свидетельствует о готовности генератора импульсного напряжения к работе - сама лампа L1 уже нагрелась и можно запускать генератор. Красный цвет светодиода HL1 констатирует рабочий режим генератора, и на лампу L1 (импульсный тиратрон) организуется поступление управляющих или поджигающих импульсов, которые задают последовательность формирования воздействующих высоковольтных импульсов. Для более явной, с точки зрения контроля, визуализации в разрабатываемой схеме было решено использовать LCD дисплей с наличием 16 символов в каждой из двух строк. Транзистор VT1, управляемый микроконтроллером, задает яркость подсветки LCD дисплея.

Поджигающие или управляющие импульсы, длительностью 4 мкс, формируются микроконтроллером DD1 на своем выводе PB3, в то время как его же вывод PB4 отвечает за подключение сетки импульсного тиратрона L1 к цепи формирования поджигающих импульсов. Перед тем как начать генерировать импульсы с микроконтроллера DD1, подается сигнал на оптопару DA4 (EL817) - открывается транзистор VT4 и ток протекает через обмотку реле К2. После этого разомкнутый в нормальном состоянии контакт реле К2 замыкается и подключает сетку импульсного тиратрона L1 к цепи генерации управляющих импульсов. Формируется последовательность управляющих импульсов, которые подаются на транзистор VT3 и через разделительный трансформатор TV3 (1:1) поступают на микросхему DD3 (IR4428), управляющую полевым транзистором VT2. Транзистор VT2, в свою очередь, увеличивает амплитуду этих импульсов до 200 В, которые через разделительный трансформатор TV4 поступают в блок коммутатора на сетку импульсного тиратрона L1.

II. Блок коммутатора. Напряжение 220 В из сети переменного тока подводится к первичной обмотке понижающего трансформатора TV5 (220/6,3), со вторичной обмотки которого напряжение 6,3 В подается уже на накал импульсного тиратрона L1. Для предотвращения ложного срабатывания, которое характеризуется открытием лампы L1, то есть до того момента времени, когда управляющие импульсы еще не поступают, сетка лампы электрически соединена с общей для всей схемы клеммой «земля» через резистор R19, в результате чего отключена от цепи формирования поджигающих импульсов в блоке управления. Для недопущения подачи на сетку импульсного тиратрона L1 постоянного напряжения, которое бы не давало ей закрыться, было предложено смонтировать в схеме 
конденсатор C15. В тот момент времени, когда уже организована подача управляющих импульсов от блока управления, последние попадают через конденсатор С15 на сетку лампы L1и открывают тем самым импульсный тиратрон. После этого электрическая емкость выходного каскада C16 разряжается на нагрузку RH, в которой преобладает активный характер, формируя тем самым высоковольтный импульс с крутым фронтом и экспоненциальным срезом (рисунок 2). Следует учитывать тот факт, что импульсный тиратрон L1 может быть закрыт только при наличии на аноде напряжения не более $300 \mathrm{~B}$.

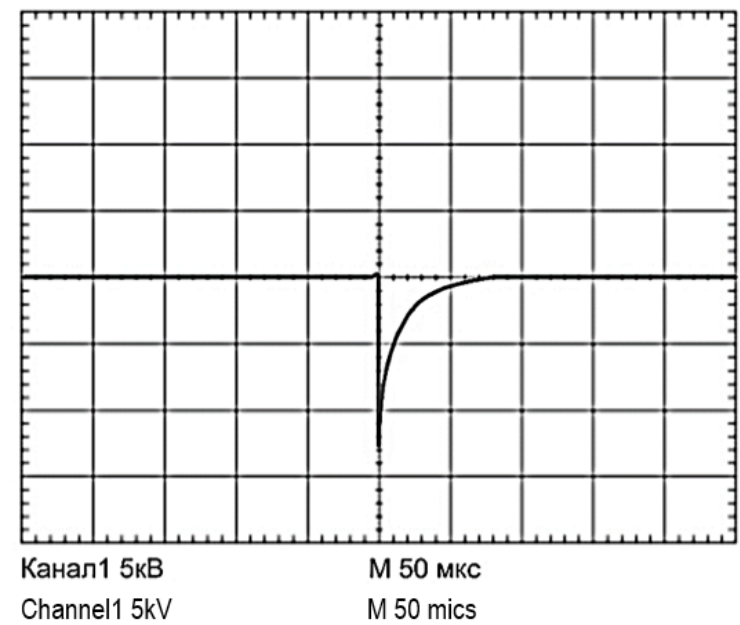

Рисунок 2 - Осциллограмма высоковольтного импульса: амплитуда - 13 кВ, длительность нарастания фронта - 300 нс; длительность экспоненциального среза - 63 мкс, длительность импульса - 65 мкс

Figure 2 - Oscillogram of high-voltage impulse: amplitude is $13 \mathrm{kV}$, rise time of the front is $300 \mathrm{~ns}$; exponential cutoff duration is $63 \mu \mathrm{s}$, impulse duration is $65 \mu \mathrm{s}$

III. Высоковольтный блок. Основная его функциональная задача - обеспечивать зарядку электрической емкости выходного каскада С16. Основными элементами этого блока являются: защитный предохранитель FU2; автотрансформатор TV6; повышающий трансформатор TV7 (ТВИ 15/20); токоограничивающий резистор на высокое напряжение R20; высоковольтный выпрямительный полупроводниковый диод VD7 и накопительный конденсатор выходного (разрядного) каскада C16. Для плавного регулирования напряжения на первичной обмотке повышающего трансформатора TV7 применяется лабораторный автотрансформатор TV6, а для получения выпрямленного высокого напряжения - диод VD7. Для ограничения тока заряда накопительного конденсатора выходного каскада С16 используют резистор R20.

IV. Нагрузка. Нагрузкой Rн генератора импульсного напряжения может быть любой исследуемый объект, подвергаемый электроимпульсному воздействию для достижения технологического эффекта. В рассматриваемом случае при настройке в качестве нагрузки использовалась как однородная масса почвы, так и почва, содержащая диэлектрические включения, которая помещалась в рабочую ячейку для обработки. Личинки саранчи моделировались за счет применения диэлектрических включений полипропиленовых предметов овальной продолговатой формы, по своим очертаниям и размерам напоминающим кубышки насекомых. Рабочая ячейка, по своей функциональной нагрузке соответствующая плоскому конденсатору, изготовлена из листового диэлектрического материала в виде прямоугольного параллелепипеда и двумя плоско- 
параллельными пластинчатыми электродами из нержавеющей стали, между которыми в воздушном объеме размещались почвенные образцы для обработки. Электроды размещались в ячейке таким образом, что нижний электрод неподвижно закреплен на дне корпуса, а верхний, подпружиненный, выполнен с возможностью перемещаться по высоте, создавая фиксированное давление на почвенный массив.

Результаты и обсуждение. Спроектированный генератор импульсного напряжения был изготовлен, прошел пуско-наладочные испытания и проверен в работе, показал в условиях лаборатории высокую стабильность формирования высоковольтных импульсов на выходе и надежность функционирования в составе экспериментального исследовательского комплекса (рисунок 3) по изучению влияния электроимпульсных воздействий на почвенные образцы.

Исследовательский комплекс для проведения лабораторных экспериментов включает в себя следующие аппараты и установки: 1) лабораторный автотрансформатор; 2) повышающий высоковольтный испытательный трансформатор, типа ТВИ 15/20; 3) контрольно-измерительный цифровой мультиметр, типа МТX 3282; 4) конденсатор на высокое напряжение выходного (разрядного) каскада генератора; 5) измерительный прецизионный LCR-метр, типа LCR-820; 6) блок управления коммутатором генератора импульсного напряжения; 7) блок коммутатора; 8) цифровой осциллограф с функцией запоминания как массива данных, так и графических зависимостей; 9) рабочая ячейка с личинками, находящимися в почвенных объемах.

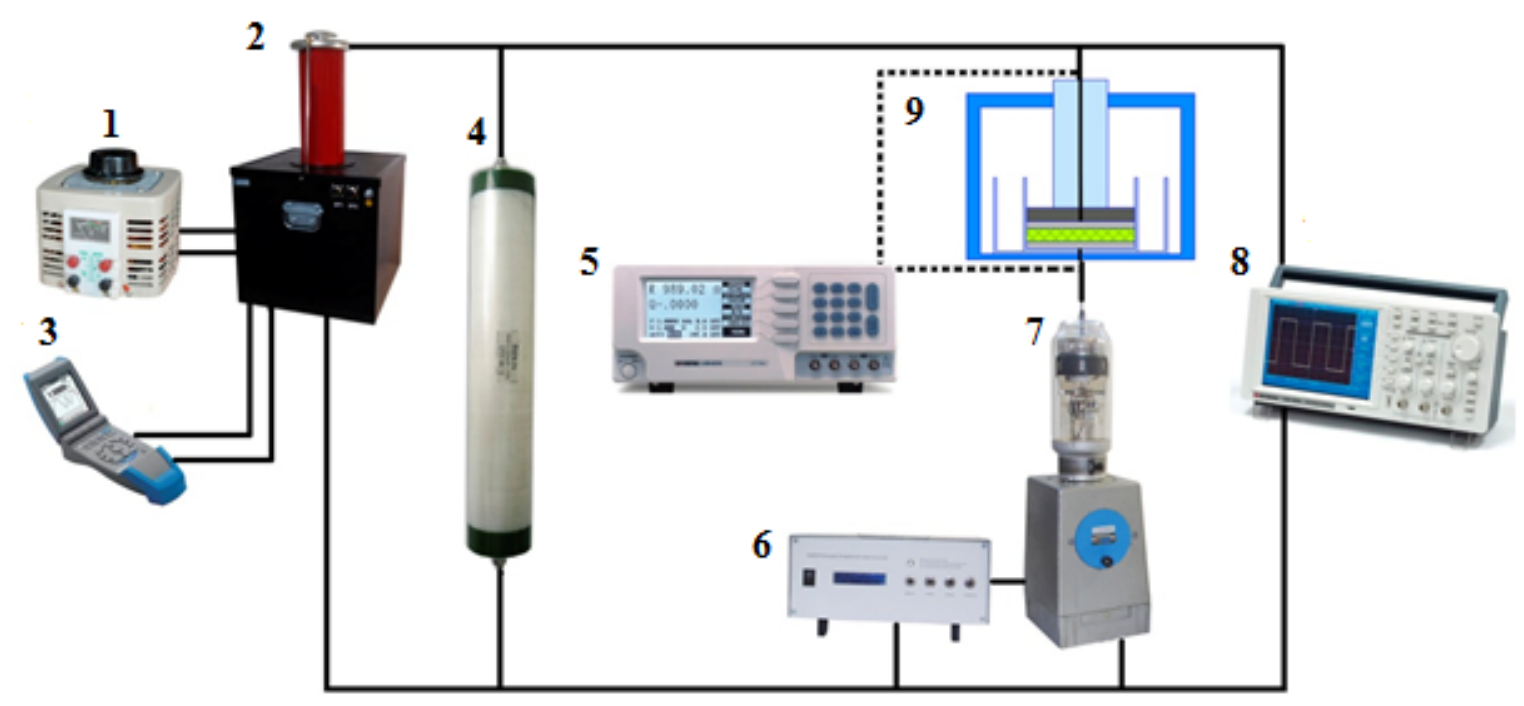

Рисунок 3 - Схема экспериментального исследовательского комплекса для изучения влияния электроимпульсных воздействий на жизнеспособность личинок саранчи:

1 - лабораторный автотрансформатор; 2 - трансформатор высоковольтный испытательный; 3 - цифровой мультиметр; 4 - высоковольтный конденсатор;

5 - прецизионный LCR-метр; 6 - блок управления коммутатором; 7 - блок коммутатора; 8 - цифровой осциллограф; 9 - рабочая ячейка с личинками, находящимися в почвенных объемах

Figure 3 - Diagram of experimental research complex for studying the effect of electrical impulse impact on the viability of locust larva:

1 - laboratory autotransformer; 2 - high-voltage test transformer; 3 - digital multimeter; 4 - high voltage capacitor; 5 - precision LCR-meter; 6 - switch control unit; 7 - switch block; 8 - digital oscilloscope; 9 - working cell with larva in soil volumes 
Рабочая ячейка, в которой осуществляется обработка почвенных образцов, представляет собой конструкцию в виде параллелепипеда с двумя электродами, расположенными в ее основании, и в регулируемой по высоте подвижной крышке. Некоторый объем почвы размещается в межэлектродном пространстве ячейки, прижимаясь с некоторым усилием верхним электродом. Перед началом электроимпульсной высоковольтной обработки LCR-метр подключается к силовым зажимам электродов и производится измерение начального сопротивления почвенного образца. После проведения измерений LCR-метр отключается от контактов силовой цепи и к ним подключается выходной блок генератора импульсного напряжения - высоковольтный трансформатор, конденсатор выходного каскада генератора и коммутатор. Используя органы регулирования на передней панели блока управления коммутатором, задаем необходимую по технологическим требованиям частоту повторения воздействующих высоковольтных импульсов и фиксируем их требуемое, по задачам эксперимента, количество. Напряжение, необходимое для заряда конденсатора выходного (разрядного) каскада генератора, устанавливается с помощью лабораторного автотрансформатора с фиксацией показаний по цифровому мультиметру, который подключен к резистивному делителю высоковольтного трансформатора. Сам процесс электроимпульсной обработки протекает следующим образом: лабораторный автотрансформатор подключается к сетевому напряжению промышленной частоты и устанавливается соответствующее экспериментальным задачам значение напряжения на вторичной обмотке высоковольтного трансформатора - на панели блока управления с помощью органов управления выставляется необходимая частота повторения высоковольтных импульсов и ограничивается их количество - блок управления осуществляет управление блоком коммутатора и с заданными параметрами разряжает конденсатор выходного (разрядного) каскада генератора на почвенный объем, находящийся в рабочей ячейке. После завершения процесса электроимпульсной обработки силовые зажимы рабочей ячейки отключаются от высоковольтной цепи и присоединяются к измерительной, чтобы при помощи LCR-метра измерить изменившееся в процессе обработки сопротивление почвенного образца. Цифровой осциллограф фиксирует характер изменения и количественные показатели напряжения и тока, а опытные данные сохраняются в персональном компьютере.

Спроектированная исследовательская установка, собранная с основным компонентом - генератором импульсного напряжения, позволяет, в зависимости от задаваемого амплитудного значения напряжения обработки и емкости конденсатора выходного каскада, реализовывать следующие задачи и действия:

1) устанавливать в соответствии с предварительно заданным алгоритмом частоту повторения (следования) высоковольтных импульсов, верхняя граница которых определяется предварительно заданным значением, а нижняя - равна частоте 1 Гц;

2) устанавливать и контролировать в соответствии с предварительно заданным алгоритмом при помощи счётчика количество воздействующих высоковольтных импульсов;

3) генерировать в соответствии с предварительно заданным алгоритмом высоковольтные импульсы напряжения амплитудой до 16 кВ, имеющие экспоненциальный срез и различную длительность.

Высоковольтный импульс напряжения имеет крутой фронт вследствие быстрого отпирания тиристора (0,3 мкс), а пологий срез - из-за долгого разряда конденсатора выходного каскада на резистивную нагрузку. Энергия, запасенная в электрическом поле конденсатора выходного каскада, при разряде полностью поглощается обрабатываемым образцом почвы, размещенным в межэлектродном пространстве рабочей ячейки. 
При наладке и проверке работоспособности разработанной конструкции генератора импульсного напряжения, проводилось осциллографирование формируемых на его выходе импульсов напряжения и тока (рисунок 4), протекающего через почвенный образец, размещенный в рабочей камере между электродами. Анализ снятых осциллограмм позволяет предположить, что почвенный объем представляет собой практически активную нагрузку, что следует учитывать при проведении натурных экспериментов.

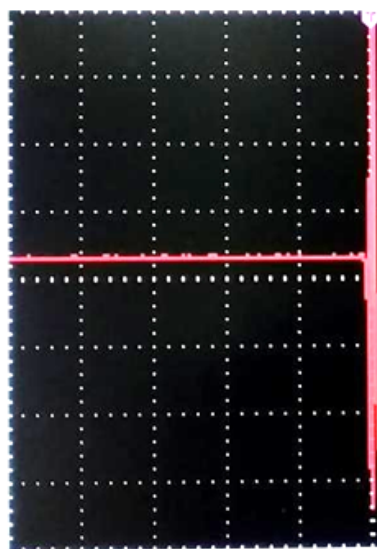

Канал1 10кB

Channel1 $10 \mathrm{KV}$

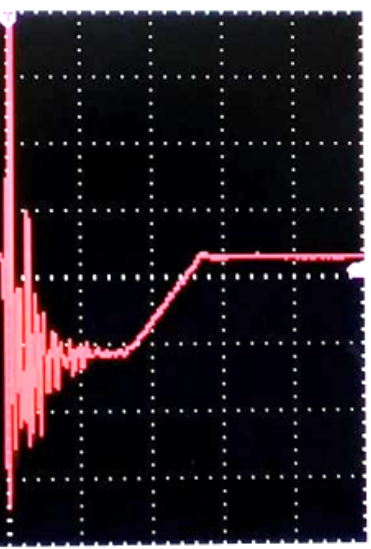

M $500 \mathrm{HC}$

M $500 \mathrm{~ns}$

a)

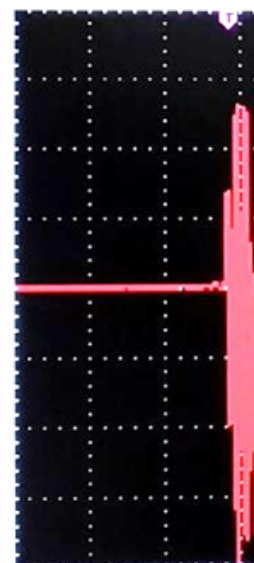

Канал1 10A

Channel1 10A

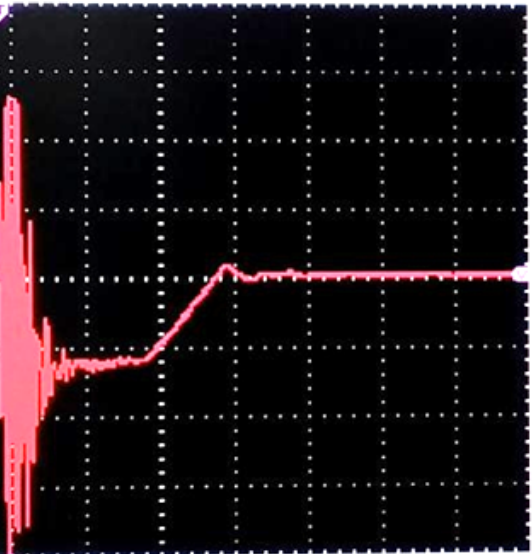

M $500 \mathrm{HC}$

M $500 \mathrm{~ns}$

б)

Рисунок 4 - Осциллограммы воздействующего импульса напряжения (а) и импульса тока (б), протекающего через обрабатываемый почвенный объем.

Сопротивление почвенного объема в рабочей камере, измеренное на частоте 10 кГц -47,51 кОм;

а на постоянном токе $-81,48$ кОм

Figure 4 - Oscillograms of the acting voltage impulse (a) and the current pulse (b), flowing through the cultivated soil volume.

Resistance of the soil volume in the working chamber, measured at frequency of $10 \mathrm{kHz}$ is $47.51 \mathrm{kOhm}$; and on direct current it equals to $81.48 \mathrm{kOhm}$

Выводы. Спроектированный и изготовленный генератор импульсного высокого напряжения при испытаниях в составе экспериментального комплекса показал высокую надежность и позволил получить требуемые заданием экспериментальных исследований параметры формируемых импульсов. Исследовательский комплекс, собранный на основе генератора импульсного напряжения, позволяет в лабораторных условиях не только осуществлять обработку почвенных образцов, но и дает возможность в функции непрерывного сканирования с помощью компьютера обрабатывать результаты экспериментов, внося коррективы в обработку и оценивая получаемые результаты. Небольшие коррективы, которые предполагается внести в схему и которые касаются ее питания, позволят исследовательский комплекс применять и при проведении полевых экспериментов.

\section{Библиографический список}

1. Вужицкий А. В., Куценко Ю. Н. Анализ методов и электрофизических устройств для уничтожения прикорневых вредителей в садоводстве // Вестник аграрной науки Дона. 2012. № 2 (18). C. 47-53.

2. Королев В. А., Топорков В. Н. Интенсификация электроимпульсных процессов в агротехнологиях // Вестник аграрной науки Дона. 2017. № 3. С. 5-10. 
3. Перспективные способы борьбы с саранчой / Г. В. Никитенко, А. А. Лысаков, Е. В. Коноплев [и др.] // Сельский механизатор. 2019. № 11. С. 12-13.

4. Топорков В. Н., Королев В. А. Электроимпульсная установка для борьбы с сорняками: монография. М.: ФНАЦ ВИМ, 2017. 132 с.

5. Юдаев И. В. Технологически эффективная и экологически безопасная борьба с сорными растениями // Сельский механизатор. 2020. № 8. С. 2-3.

6. Baev V. I., Yudaev I. V. Efficiency estimation of type of the electrical exposure on plants at their processing // AD ALTA-Journal of Interdisciplinary Research. 2018. Vol. 8(1). Pp. 252-257.

7. Cost Effective Design of High Voltage Impulse Generator and Modeling in Matlab / Z. Javid, Ke-J. Li, K. Sun [et. al.] // J Electr Eng Technol. 2018. № 13 (3). Pp. 1346-1354.

8. Design and Construction of $30 \mathrm{kV}$ High Voltage Generator using Fly-back Converter / A. Singhasathein, W. Kesil, S. Boonyayut [et. al.] // Applied Mechanics and Materials. 2015. Vol. 781. Pp. 361-365.

9. Effect of electric pulse charged to culture soil on improvement of nutritional soil condition and growth of lettuce (Lactuca sative L.) / J. Y. Yi, J. W. Choi, B. Y. Jeon [et. al.] // Agricultural Sciences. 2012. № 3. Pp. 941-948.

10. Electrotechnology as One of the Most Advanced Branches in the Agricultural Production Development / V. I. Baev, V. A. Petrukhin, I. V. Baev [et. al.] // Handbook of Research on Renewable Energy and Electric Resources for Sustainable Rural Development (ed. by V. Kharchenko and Pandian Vasant). Hershey PA: IGI Global, 2018. Pp.283-310.

11. Kirthy Reddy M., Penchalaraju M. Pulsed electric field technology in food processing industry-a review // International journal of science and research. 2014. Vol. 3. Pp.1144-1149.

12. Plant tissue sensitivity to electrical impulse / I. V. Yudaev, Y. V. Daus, V. V. Gamaga [et. al.] // Research Journal of Pharmaceutical, Biological and Chemical Sciences. 2018. Vol. 9 (4). Pp. 734-739.

13. Pulsed electric field assisted vacuum freeze-drying of apple tissue / O. Parniakov, O. Bals, N. Lebovka [et. al.] // Innovative food science \& emerging technologies. 2016. № 35. Pp. 52-57.

14. Stankovic M. V., Cvijanovic M., Dukic V. Ecological importance of electrical devices innovative in the process of anti Ambrosia artemisiifolia L. // Economics of Agriculture. 2016. Vol. 3. Pp. 861-870.

15. The effects of electric, magnetic and electromagnetic fields on microorganisms in the perspective of bioremediation / G. Beretta, A. F. Mastorgio, L. Pedrali [et. al.] // Rev Environ Sci Biotechnol. 2019. № 18. Pp. 29-75.

16. Ur-Rehman A., Khan N. Design and Fabrication of a High Voltage Lightning Impulse Generator // Engineering. 2016. № 8. Pp. 69-73.

Conclusions. The designed and manufactured high-voltage pulse generator during tests as part of the experimental complex showed high reliability and allowed us to obtain the parameters of the generated pulses required by the task of experimental studies. The research complex, assembled on the basis of a pulse voltage generator, allows not only to process soil samples in laboratory conditions, but also makes it possible to process the results of experiments in the function of continuous scanning with the help of a computer, making adjustments to the processing and evaluating the results obtained. Small adjustments that are supposed to be made to the scheme and which relate to its nutrition will allow the research complex to be used in conducting field experiments.

\section{References}

1. Vuzhickij A. V., Kucenko Yu. N. Analiz metodov i ]lektrofizicheskih ustrojstv dlya unichtozheniya prikornevyh vreditelej v sadovodstve // Vestnik agrarnoj nauki Dona. 2012. № 2 (18). S. 47-53.

2. Korolev V. A., Toporkov V. N. Intensifikaciya ]lektroimpul'snyh processov v agrotehnologiyah // Vestnik agrarnoj nauki Dona. 2017. № 3. S. 5-10.

3. Perspektivnye sposoby bor'by s saranchoj / G. V. Nikitenko, A. A. Lysakov, E. V. Konoplev [i dr.] // Sel'skij mehanizator. 2019. № 11. S. 12-13. 
4. Toporkov V. N., Korolev V. A. \}lektroimpul'snaya ustanovka dlya bor'by s sornyakami: monografiya. M.: FNAC VIM, 2017. $132 \mathrm{~s}$.

5. Yudaev I. V. Tehnologicheski ]ffektivnaya i ]kologicheski bezopasnaya bor'ba s sornymi rasteniyami // Sel'skij mehanizator. 2020. № 8. S. 2-3.

6. Baev V. I., Yudaev I. V. Efficiency estimation of type of the electrical exposure on plants at their processing // AD ALTA-Journal of Interdisciplinary Research. 2018. Vol. 8(1). Pp. 252-257.

7. Cost Effective Design of High Voltage Impulse Generator and Modeling in Matlab / Z. Javid, Ke-J. Li, K. Sun [et. al.] // J Electr Eng Technol. 2018. № 13 (3). Pp. 1346-1354.

8. Design and Construction of $30 \mathrm{kV}$ High Voltage Generator using Fly-back Converter / A. Singhasathein, W. Kesi1, S. Boonyayut [et. al.] // Applied Mechanics and Materials. 2015. Vol. 781. Pp. 361-365.

9. Effect of electric pulse charged to culture soil on improvement of nutritional soil condition and growth of lettuce (Lactuca sative L.) / J. Y. Yi, J. W. Choi, B. Y. Jeon [et. al.] // Agricultural Sciences. 2012. № 3. Pp. 941-948.

10. Electrotechnology as One of the Most Advanced Branches in the Agricultural Production Development / V. I. Baev, V. A. Petrukhin, I. V. Baev [et. al.] // Handbook of Research on Renewable Energy and Electric Resources for Sustainable Rural Development (ed. by V. Kharchenko and Pandian Vasant). Hershey PA: IGI Global, 2018. Pp.283-310.

11. Kirthy Reddy M., Penchalaraju M. Pulsed electric field technology in food processing industry-a review // International journal of science and research. 2014. Vol. 3. Pp.1144-1149.

12. Plant tissue sensitivity to electrical impulse / I. V. Yudaev, Y. V. Daus, V. V. Gamaga [et. al.] // Research Journal of Pharmaceutical, Biological and Chemical Sciences. 2018. Vol. 9 (4). Pp. 734-739.

13. Pulsed electric field assisted vacuum freeze-drying of apple tissue / O. Parniakov, O. Bals, N. Lebovka [et. al.] // Innovative food science \& emerging technologies. 2016. № 35. Pp. 52-57.

14. Stankovic M. V., Cvijanovic M., Dukic V. Ecological importance of electrical devices innovative in the process of anti Ambrosia artemisiifolia L. // Economics of Agriculture. 2016. Vol. 3. Pp. 861-870.

15. The effects of electric, magnetic and electromagnetic fields on microorganisms in the perspective of bioremediation / G. Beretta, A. F. Mastorgio, L. Pedrali [et. al.] // Rev Environ Sci Biotechnol. 2019. № 18. Pp. 29-75.

16. Ur-Rehman A., Khan N. Design and Fabrication of a High Voltage Lightning Impulse Generator // Engineering. 2016. № 8. Pp. 69-73.

\section{Information about the authors}

Yudaev Igor Viktorovich - Doctor of Technical Science, professor, professor of the Power Supply of Enterprises and Electrotechnology department, vice-rector for academic and educational work of FSBEI HE "St. Petersburg State Agrarian University". Phones: +7-902-381-83-39. E-mail: etsh1965@mail.ru.

ORCID: http://orcid.org/0000-0002-3435-4873

Eviev Valery Andreevich-Doctor of Technical Sciences, associate professor, professor of the Agricultural Engineering department, dean of the Engineering and Technology faculty of FSBEI HE "Kalmyk State University named after B. Gorodovikov". Phone: 8 (84722)3-45-49, e-mail: aviev@yandex.ru

Belyaeva Balyuta Irendenevna-Candidate of Pedagogical Sciences, associate professor of the Agricultural Engineering department of the Engineering and Technology faculty of FSBEI HE "Kalmyk State University named after B. Gorodovikov". Phone: 8 (84722)3-45-49, e-mail: belva67@mail.ru

Kokurin Ruslan Gennadievich - postgraduate student of the Heat Power Engineering and Information Control Systems department, Azov-Black Sea Engineering Institute FSBEI HE Don SAU. Phone: + 7-999626-78-10, e-mail: chemistr@yandex.ru

\section{Информация об авторах}

Юдаев Игорь Викторович, доктор технических наук, профессор, профессор кафедры «Энергообеспечение предприятий и электротехнологии», проректор по учебной и воспитательной работе ФГБОУ ВО «Санкт-Петербургский государственный аграрный университет» (196601 г. СанктПетербург, г. Пушкин, Петербургское шоссе, дом 2) Телефон: +7-902-381-83-39. Еmail:etsh1965@mail.ru. ORCID: http://orcid.org/0000-0002-3435-4873 
Эвиев Валерий Андреевич, доктор технических наук, доцент, профессор кафедры агроинженерии, декан инженерно-технологического факультета Калмыцкого государственного университета имени Б. Б. Городовикова (Республика Калмыкия, г. Элиста, ул. Пушкина, 11). E-mail:aviev@yandex.ru

Беляева Балюта Иренденевна, кандидат педагогических наук, доцент кафедры агроинженерии инженерно-технологического факультета Калмыцкого государственного университета имени Б. Б. Городовикова (Республика Калмыкия, г. Элиста, ул. Пушкина, 11). E-mail: belva67@mail.ru

Кокурин Руслан Геннадьевич, аспирант кафедры «Теплоэнергетика и информационноуправляющие системы», Азово-Черноморского инженерного института ФГБОУ ВО Донской ГАУ (347740, Ростовская область, г. Зерноград, ул. Ленина, д. 21). E-mail: chemistr@yandex.ru

\title{
DOI: 10.32786/2071-9485-2021-02-46 \\ DYNAMICS OF PRECIPITATION AND TEMPERATURE IN THE SUMMER PERIOD ON THE TERRITORY OF THE VOLGOGRAD TRANS-VOLGA REGION
}

\author{
V.G. Yuferev, N.A. Tkachenko, \\ Federal State Budget Scientific Institution \\ «Federal Scientific Centre for Agroecology, Complex Melioration and protective \\ Afforestation of the Russian Academy of Sciences», Volgograd
}

Received 10.02.2021

Submitted 11.05.2021

\begin{abstract}
The work is executed at financial support of the RFBR and the Volgograd oblast in the framework of the scientific project No. 19-45-340003 "Scientific justification and patterns of state change of landscapes ecotone Small Sirt - Caspian lowland in Volgograd Trans-Volga region".
\end{abstract}

Summary

A significant part of the lands of the Volgograd Trans-Volga region, which are in agricultural circulation, is located in the zone of unstable and insufficient moisture. As a result, the determination of the dynamics of precipitation and temperature, the characteristics of their changes are important for the forecast of droughts. Analysis of spatial data on changes in climatic conditions has shown that the dynamics tends to tighten the arid conditions and increase the likelihood of very severe droughts in the Volgograd Trans-Volga region.

Abstract
The relevance of research on the territory of the Volgograd Trans-Volga region is determined by the prolonged absence of precipitation or their significant reduction in comparison with the average longterm norms in unfavorable years, which, in conditions of high temperatures of air masses, the upper layer of soil and prolonged winds, leads to drought, in which the humidity of air and soil sharply decreases, the water balance of vegetation is disturbed, the productivity of agricultural products decreases. Introduction. A significant part of the lands of the Volgograd Trans-Volga region, which are in agricultural circulation, are located in the zone of unstable and insufficient moisture. As a result, the determination of the dynamics of precipitation and temperature, the characteristics of their change are important for the forecast of droughts. Methods and techniques. The analysis of spatial data on changes in climatic conditions was carried out using the methods of geostatistics, as well as geographic information systems for the spatial determination of the average values of the main meteorological quantities for the period 1969-2020. Dynamics studies were carried out in the period from April to October. As a result, changes in the amount of precipitation, the amount of average daily temperatures and changes in the Selyaninov hydrothermal coefficient were established. Results and conclusion. Studies have shown that the dynamics of changes in the amount of precipitation for the period under consideration is determined by the average speed $V_{R}=-1,88 \mathrm{~mm}$ per year and shows a decrease in precipitation in the period under consideration by $96 \mathrm{~mm}$. The dynamics of the sum of average daily temperatures is determined by the average rate $\mathrm{Vt}=0,32 \%$ year and shows an increase in the sum of average daily temperatures in the period under consideration by $16^{\circ}$. The average rate of decrease in Selyaninov's hydrothermal coefficient is 0.001 units per year. For 50 years, on the territory of the Volgograd Trans-Volga region, Selyaninov's hydrothermal coefficient has decreased by 0.05 units. A tendency has been established to toughen arid conditions and the likelihood of very severe droughts on 